\title{
History and Significance of the Morton Number in Hydraulic Engineering
}

\section{Michael Pfister}

Research and Teaching Associate, Laboratory of Hydraulic Constructions (LCH), Ecole Polytechnique Fédérale de Lausanne (EPFL), Station 18, CH-1015 Lausanne, Switzerland (corresponding author). E-mail: michael.pfister@epfl.ch

\section{Willi H. Hager, F.ASCE}

Professor, Laboratory of Hydraulics, Hydrology and Glaciology (VAW), ETH Zurich, Wolfgang-Pauli-Str. 27, CH-8093 Zürich, Switzerland. E-mail: hager@vaw.baug.ethz.ch

Forum papers are thought-provoking opinion pieces or essays founded in fact, sometimes containing speculation, on a civil engineering topic of general interest and relevance to the readership of the journal. The views expressed in this Forum article do not necessarily reflect the views of ASCE or the Editorial Board of the journal.

DOI: 10.1061/(ASCE)HY.1943-7900.0000870

\section{Introduction}

Two-phase air-water flows occur frequently in hydraulic engineering. Their characteristics, particularly the depth-averaged air concentrations, are of relevance for design. These must be known, for instance, to provide an adequate chute freeboard, or to avoid chocking of steep tunnel flows. Currently, two main tools are available to estimate the two-phase flow characteristics: physical modeling and numerical simulations. Both are, however, subjected to uncertainties. Physical models are prone to scale effects, whereas numerical simulations are sensitive regarding the code, calibration, as well as the validation of the results.

A correct reproduction of the air-water flow properties is difficult, given that simultaneous modeling of the dominant forces is impossible if water is used both in the prototype and the model. For bubbles, these forces are expressed with the Weber $\mathbf{W}_{b}$, Reynolds $\mathbf{R}_{b}$, and Froude $\mathrm{F}_{b}$ bubble numbers, as demonstrated by Schmidt (1934a). His fundamental work and the validation by Haberman and Morton (1953) indicate that the fluid (described by the nondimensional parameter $\mathrm{M}$ ) affects the bubble features, at least as long as $\mathrm{W}_{b}$ and $\mathrm{R}_{b}$ are below a certain value. A similar observation was made for mixture air-water flows. Accordingly, scale effects related to air concentrations in modeled flows remain, but are small if these limits are respected. The parameter $\mathrm{M}$, namely, the Morton number, is thus a key variable in modeling air-water two-phase flows.

This forum paper identifies initial sources proposing the Morton number, focusing on the individual bubble behavior in a stagnant fluid. Related results are then transferred in the framework of an analogy to the general modeling of high-speed two-phase airwater flows. These approaches are detailed based on $\mathrm{M}$, although this number was proposed for different conditions. Finally, short biographies of the three main persons involved in the development of $\mathrm{M}$ are presented, namely, those of Ernst Schmidt, William L. Haberman, and of Rose K. Morton-Sayre.

\section{Investigations on Bubble Rise Velocity}

\section{Dimensional Analysis}

The motion of gas bubbles in boiling water was of particular relevance in the early 19th century, as it affects the water circulation in boilers of steamboats, steam engines, and pumps. Mechanical engineers focused on the latter to optimize their efficiency. Using simplified approaches with stagnant water intended to allow for analytical approaches, which were not successful according to Schmidt (1934a), however. He conducted a dimensional analysis of the bubble motion in stagnant fluids, finding that the related processes may be described with the following dimensionless numbers

$$
\mathrm{R}_{b}=\frac{V_{b} D}{\nu}
$$

$$
\mathrm{F}_{b}=\frac{V_{b}}{\sqrt{g D}}
$$

$$
\mathbf{W}_{b}=\frac{\rho V_{b}^{2} D}{\sigma}
$$

Here, $g=$ gravity acceleration, $v=$ kinematic viscosity, $\rho=$ density, $\sigma=$ surface tension, $V=$ velocity, $D=$ bubble diameter, and subscript $b$ refers to a bubble. Schmidt mentioned that the bubble (1) Reynolds number $\mathrm{R}_{b}$ describes the ratio of inertia to viscous forces, (2) Froude number $F_{b}$ the ratio of inertia to gravity, and (3) Weber number $W_{b}$ the ratio of inertia to surface forces. However, Eqs. (1)-(3) do not allow for explicitly deriving $V_{b}$. Schmidt $(1934 a, b)$ proposed to rearrange the equations to provide only one explicit term containing $V_{b}$, namely, $\mathrm{R}_{b}$ according to Eq. (1). The other two terms are then

$$
\begin{gathered}
\frac{\mathbf{W}_{b}}{\mathrm{~F}_{b}^{2}}=\frac{D^{2} \rho g}{\sigma} \\
\frac{\sqrt[3]{\mathrm{F}_{b}^{2} \mathrm{R}_{b}^{4}}}{\mathbf{W}_{b}}=\frac{\sigma}{\rho \sqrt[3]{g \nu^{4}}}
\end{gathered}
$$

Note that Eq. (4) includes $D, g$, and fluid constants, whereas Eq. (5) consists exclusively of fluid properties and $g$. Consequently $V_{b}$ in a stagnant, infinite fluid body is explicitly expressed by nondimensional numbers and their combinations as (Schmidt 1934a, b)

$$
\frac{V_{b} D}{\nu}=f\left[\frac{D^{2} \rho g}{\sigma}, \frac{\sigma}{\rho \sqrt[3]{g \nu^{4}}}\right]
$$

Schmidt (1934a) concluded his investigation with the remark that physical experiments are required to support the above mentioned hypotheses, in particular including different fluids. 


\section{Experimental Validation}

Rosenberg (1950) presented a literature survey on the motion of air bubbles in liquids, reporting a considerable scatter and uncertainty. He thus conducted model tests to determine the latter. Based on a preliminary dimensional analysis, he derived the relevant parameters as the drag coefficient, the Reynolds number, and a third parameter involving only the fluid properties. By referring to Schmidt (1934a), Rosenberg denotes this parameter [derived from Eq. (5)] with the symbol $M$ as

$$
\mathbf{M}=\frac{g \mu^{4}}{\rho \sigma^{3}}
$$

Here, $\mu=$ dynamic viscosity. The dependence of the bubble drag, shape, and path on $\mathrm{R}_{b}$ was determined. Since the tests were confined to one single liquid only, the effect of $\mathrm{M}$ remained unascertained. The results indicated that this parameter may have a considerable effect on the motion and shape of a bubble.

Haberman and Morton (1953) continued the work of Rosenberg by conducting experiments on the drag coefficient of rising air bubbles in various stagnant fluids and under different temperatures. The following liquids were used: Water $\left(6,19,21\right.$, and $\left.49^{\circ} \mathrm{C}\right)$, glim solution, mineral oil, varsol, turpentine, methyl alcohol, olive oil, syrup, different corn syrup-water mixtures, glycerin-water mixtures, and an ethyl alcohol-water mixture. The explicit effect of $\mathrm{M}$ on the bubble rise velocity was systematically studied for $0.3 \times 10^{-11} \leq \mathrm{M} \leq 0.2 \times 10^{-2}$.

The rate of rise velocity was found to mainly depend on the liquid viscosity for spherical bubbles, and on surface tension for ellipsoidal bubbles. The drag coefficients of small spherical bubbles overlapped with these of rigid spheres, whereas a general drag reduction occurs with increased bubble size and thus larger $\mathrm{R}_{b}$ [Fig. 1(a)]. This decrease was explained with the formation of an air circulation inside the bubble. For liquids of low $\mathrm{M}\left(<10^{-3}\right)$, a minimum in the drag curve is reached at $R_{b} \cong 250$, i.e., near the transition from spherical to ellipsoidal bubble shape. For $\mathbf{R}_{b}>$ $3 \times 10^{3}$ results the constant drag coefficient $\cong 2.6$. The transition to spherical caps is completed at $\mathrm{W}_{b} \cong 20$ [Fig. 1(b)]. The drag coefficient of spherical caps is independent of bubble size (i.e., of $\mathbf{R}_{b}$ and $\mathbf{W}_{b}$ ) and has the constant value of 2.6 [as noted from Fig. 1(a)]. Hence, the fluid (in terms of M) has no more effect on the drag if limit values of $\mathbf{R}_{b}$ and $\mathrm{W}_{b}$ are attained. These main results were published in the Proceedings and Transactions ASCE (Haberman and Morton 1954, 1956).

Haberman (1956) submitted in the framework of his Ph.D. thesis a mathematical analysis of the wall effect of spheres, based on Stokes' approximation for the hydrodynamic equations of slow flows. This work was included in Haberman and Sayre (1958)Morton was meanwhile married with Sayre as family name- who considered "the problem of steady, axial translation of rigid and fluid spheres in stationary and moving viscous, incompressible fluids bounded by an infinitely long cylinder." Experimental results confirmed the theory, suggesting that the wall effect for fluid spheres was less than for the corresponding rigid spheres.

A reformulation of Eq. (5), based on M according to Eq. (7), was presented by e.g., Chesters (1975) as

$$
\mathbf{M}=\frac{g \mu^{4}}{\rho \sigma^{3}}=\frac{\mathbf{W}^{3}}{\mathbf{R}^{4} \mathbf{F}^{2}}
$$

The first source denoting $\mathrm{M}$ as the "Morton number" appears to be Clift et al. (1978), stating "We have called this group the Morton number [ ... ], although it was used prior to Haberman and Morton (1953) by Rosenberg (1950), who refers to an even earlier user.
In literature, the group is often simply referred to as the M-group or property group."

\section{Relevance for Physical Modeling}

\section{Analogy to Physical Modeling}

High-speed air-water two-phase flows are more complex than bubbles rising in stagnant water. Selected observations of the "simple" case are nevertheless interesting to describe the complex flow features. For instance, it is known from the literature that minimum Reynolds and Weber numbers (with the flow depths as reference) limit scale effects related to air concentrations in two-phase flows. A similar observation was made by Haberman and Morton (1953) concerning the drag of a single bubble. Particular outcomes of the latter work are thus conceptually transferred from the "simple" case to complex two-phase flows, knowing that these can-strictly spoken—not be applied one-to-one.

\section{Dynamic Similarity}

Physical models are scaled with the geometrical factor $\lambda$, resulting in the length ratio between a prototype (subscript $P$ ) and a model (subscript $M$ ) reference length as $\lambda=l_{P} / l_{M}$. As to the hydraulic parameters, these are "translated" from the model to the prototype with the dynamic similarity. The latter bases essentially on Isaac Newton, according to whom the sum of all forces $F$ acting on a control volume is equal to its mass $m$ times the related acceleration $a$ (i.e., inertia $I=m a$ ). To achieve dynamic similarity between the model and the prototype, the ratio of the dominant force $F$ (relative to inertia $I$ ) should consequently be identical.

The dominant gravity, surface tension, and viscous forces of two-phase air-water flow features are listed, e.g., by Schmidt (1934a). For gravity, $F=m g$, so that (with $l=$ reference length, $t=$ reference time, and $V=$ fluid velocity)

$$
\frac{I}{F}=\frac{m a}{m g}=\frac{l^{3} \rho}{l^{3} \rho} \frac{l}{t^{2}} \frac{1}{g}=\frac{l^{2}}{t^{2}} l^{2} \frac{1}{g}=\frac{V^{2}}{l g}=\mathrm{F}^{2}
$$

Gravity is the dominant force driving free surface flows, according to Froude similarity. The Froude number $F$ shall thus be identical in both the prototype and the model.

Considering surface forces $(F=l \sigma)$ as relevant, then

$$
\frac{I}{F}=\frac{m a}{l \sigma}=\frac{l^{3} \rho}{l \sigma} \frac{l}{t^{2}}=\frac{l^{2}}{t^{2}} l \frac{\rho}{\sigma}=V^{2} l \frac{\rho}{\sigma}=\mathrm{W}
$$

Accordingly, the Weber number $\mathrm{W}$ has to be identical in the prototype and the model (Weber similarity).

If viscous forces $\left(F=\mu A V h^{-1}\right.$, with $\mu=$ dynamic viscosity, $A=$ area, and $h=$ flow depth) are relevant, then

$$
\frac{I}{F}=\frac{m a}{\mu A V h^{-1}}=\frac{l^{3} \rho}{\mu l^{2}} \frac{l}{t^{2}} \frac{t}{l} l=\frac{l^{2}}{t} \frac{\rho}{\mu}=\frac{V l}{\nu}=\mathrm{R}
$$

Therefore, the Reynolds number $\mathrm{R}$ has to be identical in the prototype and the model, referred to as Reynolds similarity. Note that the reference length taken into account for high-speed twophase flows is typically the flow depth $h$ (Pfister and Chanson 2012) instead of $D$.

Combining a specific similarity with the geometrical scale factor $\lambda$ results in the scale factors used to "translate" hydraulic parameters from a model to the prototype. For Froude similarity, for instance, the scale factor for the velocity is 

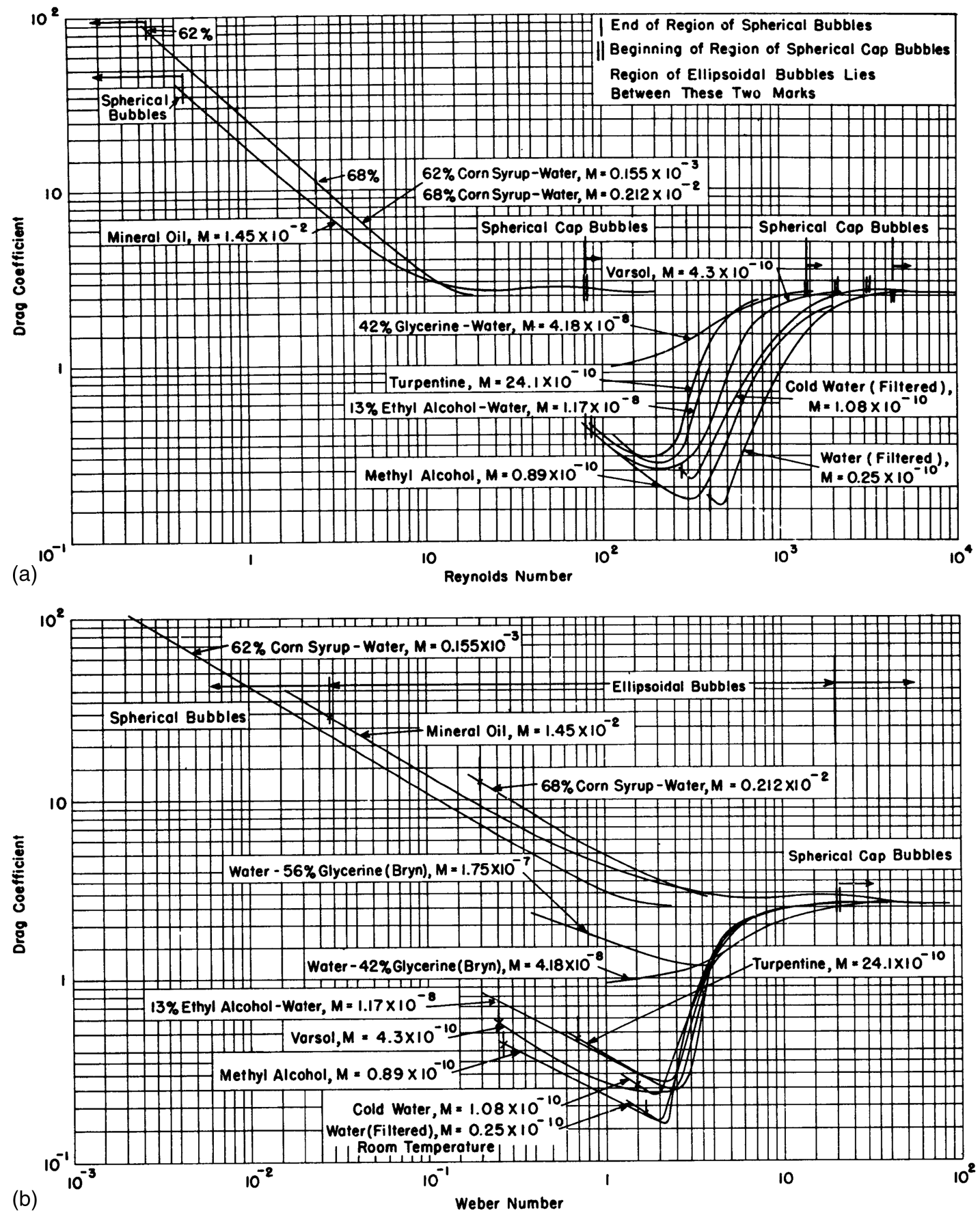

Fig. 1. Drag coefficient versus bubble: (a) Reynolds; (b) Weber numbers, for single bubble rising at terminal velocity in various stagnant fluids (Haberman and Morton 1954, (C) ASCE)

$$
\mathrm{F}_{P}=\mathrm{F}_{M} \rightarrow \frac{V_{P}}{\sqrt{g h_{P}}}=\frac{V_{M}}{\sqrt{g h_{M}}} \rightarrow \frac{V_{P}}{V_{M}}=\sqrt{\frac{g h_{P}}{g h_{M}}}=\sqrt{\lambda}
$$

These scale factors can be derived for all hydraulic parameters and similarities.

\section{Scale Effects}

The correct modeling of two-phase air-water flows requires full dynamic similarity, i.e., $\mathrm{M}, \mathrm{R}$, and $\mathrm{F}$ must be identical in both the prototype and the associated model, because all related forces affect the air entrainment and the transport features. This is, however, physically impossible, as shown below with an example.
Taking into account the Froude similarity results in a scale factor of $\lambda^{1 / 2}$ for $V$ and of $\lambda$ for $h$. Computing the prototype value of $\mathrm{R}_{P}$ based on hydraulic model data gives

$$
\mathrm{R}_{P}=\frac{\left(\sqrt{\lambda} V_{M}\right)\left(\lambda l_{M}\right)}{\nu}=\lambda^{3 / 2} \mathrm{R}_{M}
$$

In contrast, for the Weber number, $\mathrm{W}_{P}=\lambda^{2} \mathrm{~W}_{M}$. Consequently, if $\mathrm{F}_{P}=\mathrm{F}_{M}$, then $\mathrm{R}_{P} \neq \mathrm{R}_{M}$ and $\mathrm{W}_{P} \neq \mathrm{W}_{M}$. This example demonstrates that only one dynamic similarity can be respected (i.e., Froude), while the others are mandatorily violated (i.e., Reynolds and Weber). Consequently, a precise modeling with $\lambda \neq 1$ of two-phase air-water flow is physically impossible, at least 
if using water in both, prototype and model. The application of adapted fluid properties (with $\nu, \rho$, and $\sigma$ different from water) in the model allowed for a perfect dynamic similarity, but these fluids would be difficult to obtain and handle, so that this way appears not economic.

\section{Limitations of Weber or Reynolds Numbers}

An adequate reproduction of the air concentrations in two-phase model flows is nevertheless possible, as shown by Pfister and Chanson (2012), based on a literature review. To achieve reliable air concentrations, minimum values of $\mathrm{R}$ and $\mathrm{W}$ must be respected in the model. Pfister and Hager (2010), for instance, conclude that these minima are, for high-speed air-water two-phase flows using the Froude similitude, $\mathbf{W}^{0.5}>140$ or $\mathrm{R}>2.2 \times 10^{5}$, with $h$ as reference length. If respecting these minimum numbers, then the model turbulence and water surface characteristics are appropriate to allow for similar (but not identical) air concentration features as on the prototype. This approach is supported by the work of Haberman and Morton (1953): The effect of $M$ on the bubble behavior (linked to the air concentrations in models) is negligible if limit values of $\mathrm{W}_{b}$ and $\mathrm{R}_{b}$ are respected. In other words, the fluid parameters (i.e., M) have then a small effect, so that the "wrong" fluid (i.e., water) used in models has no dominant effect on the air-water flow features.

Another outcome of the works of Schmidt (1934a) and Chesters (1975) is the link between W, R, F, and M, indicating that either W or $\mathrm{R}$ must be limited to reduce scale effects. By considering only one limitation, the other is implicit. This leads to the conclusion that the limit values of $\mathrm{W}$ or $\mathrm{R}$ are relatively large for small $\mathrm{F}$, but smaller for large $F$ (Pfister and Chanson 2012). Fig. 2 illustrates the dependence of the limit $R$ on $F$, for different limit values of $\mathrm{W}^{0.5}$ and for water $\left(\mathrm{M}=3.89 \times 10^{-11}\right)$. For instance, if a physical model is operated at $F=15$, and $W^{0.5}=140$ is considered as limit value to respect, then the related limit in terms of $R=1.7 \times 10^{5}$. A model with $F=5$ and $W^{0.5}=140$ as limit value results in a limit $R=3.0 \times 10^{5}$.

\section{Biographies}

Ernst Schmidt (Fig. 3) was born on February 11, 1892 at Vögelsen, Germany, and he passed away on January 22, 1975, at Munich, Germany. He made studies of civil engineering and electrotechniques at the Universities of Dresden and Munich, obtaining

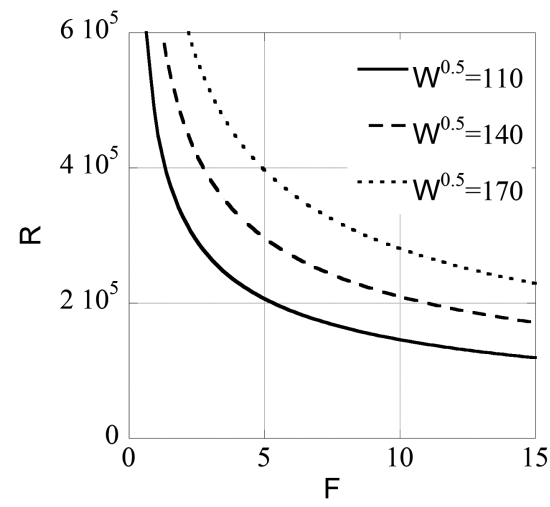

Fig. 2. Graph of Eq. (8) for water and various $W^{0.5}$ resulting in $R$ versus F (Pfister and Chanson 2012, copyright (C) International Association for Hydro-environment Engineering and Research, reprinted by permission of (Taylor \& Francis Ltd.)

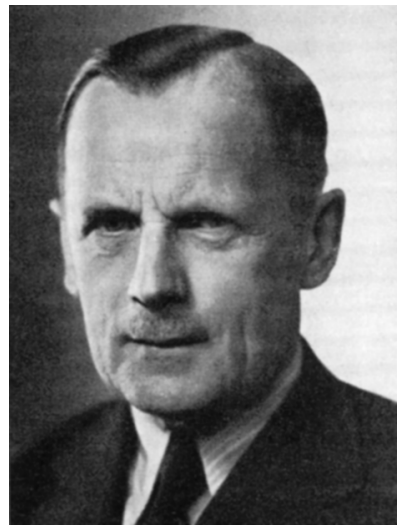

Fig. 3. Portrait of Ernst Schmidt (Hager 2009, with permission from International Association of Hydraulic Engineering and Research)

the engineering diploma in 1919. He submitted his Ph.D. thesis to the latter University, and was appointed in 1925 professor of heat flow at the University of Danzig, today's Gdansk in Poland. He accepted in 1937 the directorship of the Institute of Motor Research at the newly founded Aviation Research Center, Braunschweig, Germany. After World War II, he was appointed professor at the University of Braunschweig, moving in 1952 to the University of Munich as professor of technical thermodynamics. Schmidt was awarded honorary doctorates from the Universities of Aachen and Glasgow U.K., and received the Eucken Medal and the MaxJakob-Award. His research dealt mainly with unsteady heat conduction, the visualization of boundary layer flow, the observation of drop condensation, and the analogy between transfers of heat and matter, leading finally to the Schmidt number. His book Technische Thermodynamik was extensively used by engineers and scientists. He was also closely associated with the Verein Deutscher Ingenieure VDI, receiving the 1956 Grashof-Denkmünze as its highest honor.

William Lawrence Haberman (Fig. 4) was born on May 4, 1922 at Vienna, Austria, and passed away on December 27, 1996 at Rockville, MD, USA (Grossman Bertrand, personal communications, 2013). He emigrated to the United States with his family to escape Nazi persecution in 1939 and, shortly after entering the Cooper Union School of Engineering, New York City, in 1941, he was drafted into the U.S. Army. After serving in Burma during World War II, he reentered Cooper Union and completed a

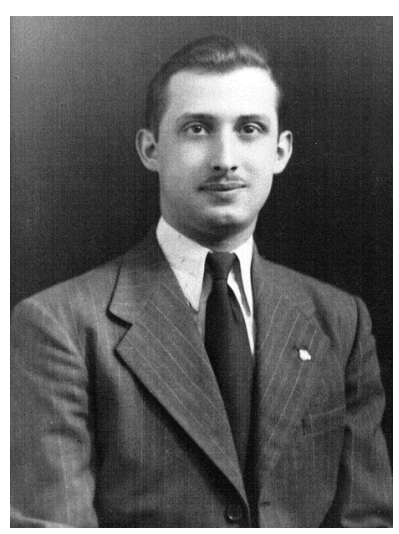

Fig. 4. Portrait of William Haberman around 1950 (photograph courtesy of Bertrand Grossman) 
Bachelor Degree in mechanical engineering in 1949. He received M.S. (1952) and Ph.D. (1956) degrees from the University of Maryland while employed at the U.S. Navy research laboratory David Taylor Model Basin (DTMB) at Bethesda, MD (Today: Naval Surface Weapons Center Carderock).

His career at DTMB spanned 15 years, including 2 years as director of the Advanced Planning Division of the Office of Naval Research. In 1964, as the effort to land a man on the moon was just getting underway, he began a seven year career with the Manned Space Flight Program as a senior staff scientist at the National Aeronautics and Space Administration (NASA) headquarters, Washington, DC.

Haberman was an adjunct professor of mechanical engineering at the University of Maryland from 1958 to 1968, Chairman of the Department of Mechanical Engineering at Newark College of Engineering from 1970 to 1973, and an adjunct professor at Montgomery College at Rockville MD, from 1973 to 1978 . He co-authored two widely used engineering textbooks which have been reprinted in several languages: Introduction to Fluid Mechanics (1988) and Engineering Thermodynamics with Heat Transfer (1989). Following his retirement from government research and teaching, Haberman continued working as an engineering consultant on various projects in fluid mechanics and heat transfer until his death in 1996.

Rose Katherine Morton was born on December 03, 1925 at Albemarle, NC (Sayre 2003, Marie Sayre-Cole and Clifford Sayre, personal communications, 2013). She enrolled at the Women's College, University of North Carolina, Greensboro, NC, as a mathematician [Fig. 5(a)], graduating in 1948 with a B.A. degree. While a senior at the college, she took an exam as prerequisite for a college graduate to obtain a technical position in the U.S. government, opening the door to join the U.S. Department of the Navy at one of their research laboratories.

She started in 1948 working as a physical science aide at DTMB, the U.S. Department of the Navy, Washington DC. In 1951 she applied for an internal job opening as mathematician, thus moving to the Hydrodynamics Division of DTMB. There she performed numerical calculations on experimental data, including towing tank experiments of the pontoons and bodies of amphibian planes; and research on analytical solutions to differential equations

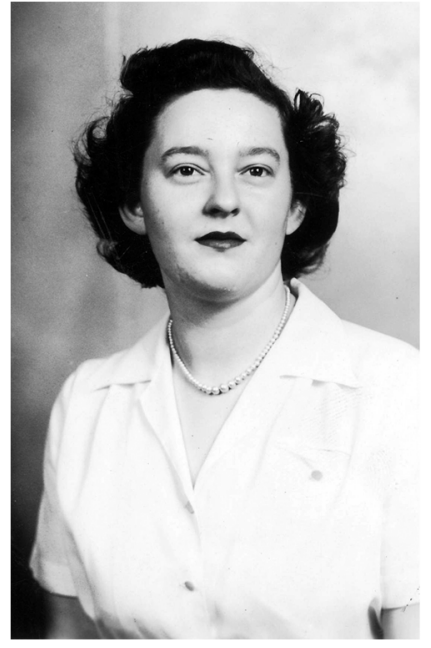

(a)

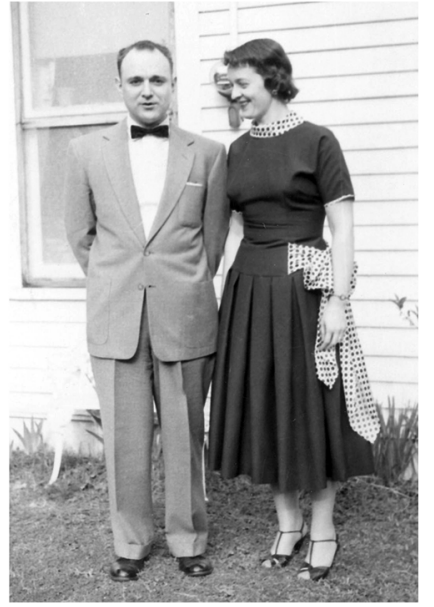

(b)
Fig. 5. (a) Portrait of Rose Morton dated 1946 (probably her college senior photo); (b) Rose Morton-Sayre with her husband Clifford (1956) (photographs courtesy of Marie Sayre-Cole and Clifford Sayre) associated with fluid motion. Of particular importance was the work done on the fluid mechanics of liquids and bubbles, collaborating with William L. Haberman. Additional projects included: amphibian plane design, naval architecture (Szebehely 1952), fluid mechanics and mines, and hydrophones.

Morton married Clifford L. Sayre, Jr., in 1953 [Fig. 5(b)], a U.S. Navy officer and mechanical engineer, also working at the Hydrodynamics Division of DTMB. Rose Morton-Sayre continued working as a mathematician at DTMB until 1958, then resigning her position to raise their children (Clifford 3rd and Marie). She passed away on November 12, 1999 at Georgetown Hospital, Washington, DC.

Hereafter, an extract of her work written in an application form for an internal job opening at DTMB is reproduced. The form is not dated, but appears to be elaborated in 1953 (Marie Sayre-Cole and Clifford Sayre, personal communications, 2013): "I am a member of the Flow Study Section of the Hydrodynamic Division. This section is chiefly concerned with the study of velocity and pressure fields including related phenomena such as cavitation, liquid-gas mixtures, and vortex-induces vibrations. My work consists primarily of mathematical analyses of all types for the theoretical and experimental phases of these problems."

"Approximately $50 \%$ of the time my duties are concerned with finding analytical solutions to differential equations of motion for various types of flow problems and with associated numerical computations. For example, in connection with a study of bubble motion in various liquids I showed that a known solution of the Navier-Stokes equation for slowly moving fluid spheres (first obtained by Hadamard 1911) is not affected by a change in the boundary conditions (i.e., the inclusion of the pressure drop across the sphere's surface due to surface tension). I am co-author of a report on the bubble investigation, currently in preparation, entitled 'The drag and shape of air bubble rising in various liquids' (Haberman and Morton 1953). Recently, I computed the trajectory of a small object placed in a potential flow about an infinite wedge. This required the use of the complex variable theory to compute flow about the wedge and a step-by-step integration of the nonlinear differential equation of motion."

"About $40 \%$ of my time is spent on numerical calculations of experimental data. I reduce raw test data to final form for presentation in reports or perform necessary computations for formulating applicable parameters for the description of the investigated physical phenomena. For example, in the study of bubble motion, I was responsible for converting the raw test data from motion picture film into the nondimensional parameters to be used in correlating the results of the tests. This included devising the best methods for reading the film, applying the necessary optical corrections to the data, and devising methods for rapid calculations of bubble size, velocity, and the desired parameters."

"The remainder of my time is spent assisting in the preparation of reports and graphs, and studying the literature pertinent to the problems in my Flow Study Section to obtain the necessary background for understanding the nature of the work assigned to me."

\section{Conclusions}

In the 1930s, the Morton number $M$ was derived from a dimensional analysis of a bubble rising in a stagnant fluid. This was of interest in the context of steam engines, and it followed that the bubble Froude $\mathbf{F}_{b}$, Weber $\mathbf{W}_{b}$, and Reynolds $\mathbf{R}_{b}$ numbers are of major influence. A rearrangement of the latter resulted in the aforementioned Morton number, including only fluid properties. In the 1950 s, a series of experiments was conducted 
to investigate the liquid effect (i.e., of $\mathrm{M}$ ) on the bubble behavior. It was concluded, among others, that the liquid affects the bubble drag if $\mathbf{W}_{b}$ and $\mathrm{R}_{b}$ are small. In parallel, $\mathrm{M}$ was expressed versus $\mathrm{F}_{b}, \mathrm{~W}_{b}$, and $\mathrm{R}_{b}$.

The air flow features in high-speed two-phase flows are more complex than in the aforementioned experiments. Nevertheless, some analogies exist and are of particular relevance for physical modeling of two-phase flows. A priori, a correct physical modeling of the air comportment in a fluid is impossible if the same fluid (i.e., with the same value of $\mathrm{M}$ ) is used both in the model and the prototype, resulting in scale effects. However, recent literature indicates that scale effects regarding air concentrations are small if respecting limit model values of $W$ and $R$, with the flow depth as the reference length. Then, the effects of viscosity and surface tension are relatively small as compared with the flow velocity and depth, allowing for a similar air concentration distribution. Another outcome linked to $\mathrm{M}$ is its expression in terms of $\mathrm{F}, \mathrm{W}$, and $\mathrm{R}$. This allows for specifying that only $\mathrm{R}$ or $\mathrm{W}$ limits have to be respected, whereas the other limit is then implicit. Accordingly, the literature approaches (some sources recommend to limit W, others R, and few both) can be combined resulting in their agreement. Finally, a dependence of the limits to considering $F$ is derived: Flows with small values of $F$ are more prone to scale effects regarding the air flow features than flows of high $\mathrm{F}$.

The added biographies illustrate the historic framework linked to the development of the Morton number. It is concluded that the latter was proposed by Ernst Schmidt, but experimentally validated by William L. Haberman and Rose K. Morton-Sayre.

\section{Acknowledgments}

The authors appreciate the support of Marie Sayre-Cole and Clifford Sayre, the children of Rose Morton-Sayre, who provided the herein given biography of their mother and the photographs. Bertrand Grossman, a nephew of William Haberman, is acknowledged for his kind assistance and the portrait. Finally, the librarians of EPFL (in particular Mr. Julien Tanari) gave an outstanding support in searching "untraceable" literature.

\section{Notation}

The following symbols are used in this paper:

$A=$ area;

$a=$ acceleration;

$D=$ bubble diameter;

$F=$ force;

$\mathrm{F}=$ Froude number;

$g$ = gravity acceleration;

$h=$ flow depth;

$I=$ inertia;

$l=$ length;

$\mathrm{M}=$ Morton number;

$m=$ mass;

$\mathrm{R}=$ Reynolds number; $t=$ time;

$V=$ velocity;

$\mathrm{W}=$ Weber number;

$\mu=$ dynamic viscosity;

$\lambda=$ scale factor;

$\nu=$ kinematic viscosity;

$\rho=$ density; and

$\sigma=$ surface tension.

\section{Subscripts}

$b=$ bubble;

$M=$ model; and

$P=$ prototype.

\section{References}

Chesters, A. K. (1975). "The applicability of dynamic-similarity criteria to isothermal, liquid-gas, two-phase flows without mass transfer." Int. J. Multiphase Flow, 2(2), 191-212.

Clift, R., Grace, J. R., and Weber, M. E. (1978). Bubbles, drops, and particles, Academic Press, New York.

Haberman, W. L. (1956). "Wall effect for rigid and fluid spheres in slow motion." Ph.D. thesis, Univ. of Maryland, College Park, MD.

Haberman, W. L., and Morton, R. K. (1953). "An experimental investigation of the drag and shape of air bubbles rising in various liquids." Rep. 802, David W. Taylor Model Basin, Bethesda, MD.

Haberman, W. L., and Morton, R. K. (1954). "An experimental study on bubbles moving in liquids." Proc. ASCE, Vol. 80(1), 1-25.

Haberman, W. L., and Morton, R. K. (1956). "An experimental study on bubbles moving in liquids." Trans. ASCE, 121, 227-252.

Haberman, W. L., and Sayre, R. M. (1958). "Motion of rigid and fluid spheres in stationary and moving liquids inside cylindrical tubes." Rep. 1143, David W. Taylor Model Basin, Bethesda, MD.

Hadamard, J. (1911). "Mouvement permanent lent d'une sphère liquide et visqueuse dans un liquide visqueux (Slow movement of a liquid and viscous sphere in a viscous liquid)." Comptes Rendus Acad. Sci. Paris, 152, 1735-1738 (in French).

Hager, W. H. (2009). Hydraulicians in Europe 1800-2000, Vol. 2, IAHR Monographs, CRC Press, Boca Raton, FL.

Pfister, M., and Chanson, H. (2012). "Discussion of scale effects in physical hydraulic engineering models." J. Hydraul. Res., 50(2), 244-246.

Pfister, M., and Hager, W. H. (2010). "Chute aerators I: Air transport characteristics." J. Hydraul. Eng., 10.1061/(ASCE)HY.1943-7900 .0000189, 352-359.

Rosenberg, B. (1950). "The drag and shape of air bubbles moving in liquids." Rep. 727, David W. Taylor Model Basin, Bethesda, MD.

Sayre, R. H. (2003). Sayre family: Another 100 years, Vol. 2, iUniverse, Lincoln, NE.

Schmidt, E. (1934a). “Ähnlichkeitstheorie der Bewegung von Flüssigkeitsgasgemischen (Similarity theory of motion in fluid-gas mixtures)." Forschungsheft, 365, VDI, Berlin, 1-3 (in German).

Schmidt, E. (1934b). "Über die Bewegungsverhältnisse von GasFlüssigkeitsgemischen (On the kinematic conditions in gas-liquid mixtures)." 72nd VDI Hauptversammlung, VDI, Berlin, 14-16 (in German).

Szebehely, V. G. (1952). "Hydrodynamics of slamming of ships." Rep. 823, David W. Taylor Model Basin, Bethesda, MD. 